\title{
INSS Stage 4
}

National Cancer Institute

\section{Source}

National Cancer Institute. INSS Stage 4. NCI Thesaurus. Code C85421.

Tumor spread to distant lymph nodes, bone marrow, bones, and liver. 